BULLETIN OF THE

AMERICAN MATHEMATICAL SOCIETY

Volume 77, Number 3, May 1971

\title{
TOEPLITZ OPERATORS IN MULTIPLY CONNECTED REGIONS
}

\author{
BY M. B. ABRAHAMSE \\ Communicated by Peter Lax, August 10, 1970
}

1. Introduction. Let $D$ be a bounded domain in the plane whose boundary consists of $n+1$ nonintersecting, analytic, Jordan curves. Let $C$ be the space of continuous complex functions on $\partial D$ and let $A$ be the subspace of $C$ consisting of those functions with continuous extensions to $\bar{D}$ which are analytic in $D$. Let $m$ be harmonic measure on $\partial D$ for some point in $D$ and let $H^{2}$ be the $L^{2}(m)$-closure of $A$. Let $P$ denote the orthogonal projection of $L^{2}(m)$ onto $H^{2}$. For $\phi$ in $L^{\infty}(m)$ the Toeplitz operator $T_{\phi}$ on $H^{2}$ is defined by $T_{\phi}(f)=P(\phi f)$ for $f$ in $H^{2}$. This paper deals with the Fredholm theory and with invertibility criteria for these operators.

On the Fredholm level, these Toeplitz operators are similar to the usual Toeplitz operators on the disk (cf. [3]). For instance, if $\phi$ is in $C$, then the essential spectrum of $T_{\phi}$ is the range of $\phi$ (Theorem 3). However, the spectrum itself may be considerably different: in this setting there are selfadjoint Toeplitz operators with eigenvalues and disconnected spectrum.

This note is primarily a statement of results and discussion. Complete proofs will appear elsewhere. The results are from my dissertation written under the direction of R. G. Douglas.

2. The algebra $H^{\infty}$. Let $H^{\infty}$ be the weak star closure of $A$ in $L^{\infty}(m)$. The following theorem is due to Tumarkin and Havinson [10] and is generalized to hypo-Dirichlet algebras by Ahern and Sarason [1].

THEOREM. If $u$ is a positive function in $L^{\infty}(m)$ and if $\log u$ is in $L^{1}(m)$, then there is a $\phi$ in $H^{\infty}$ such that $|\phi|=u$ a.e.

Now let $Y$ be the maximal ideal space of $L^{\infty}(m)$, let $\phi \rightarrow \hat{\phi}$ be the Gelfand transform from $L^{\infty}(m)$ onto $C(Y)$, and let $\hat{H}^{\infty}$ be the image of $H^{\infty}$. It is a consequence of the preceding theorem that $\hat{H}^{\infty}$ satisfies the hypotheses of the following lemma concerning function algebras.

Lemma 1. Suppose $B \subset C(X)$ is a uniform algebra whose Šilov boundary is $X$. Suppose $U$ is a nonempty open subset of $X$ and $\psi$ in $B$ does not

AMS 1970 subject classifications. Primary 47B35, 46J15; Secondary 30A78.

Key words and phrases. Toeplitz operator, Fredholm operator, Hardy spaces on multiply connected regions. 
vanish on $U$. Then there is a $\theta$ in $B$ such that $\theta \psi$ is not identically zero and $\theta \psi$ peaks on $U$.

The above theorem and the fact, proved in [1], that $H^{\infty}=H^{2}$ $\cap L^{\infty}(m)$, give the following characterization of the spectrum of $T_{\phi}$ for $\phi$ in $H^{\infty}$.

Lemma 2. Let $\phi$ be in $H^{\infty}$. Then $T_{\phi}$ is invertible if and only if $\phi$ is invertible in $H^{\infty}$.

3. The isomorphism theorem. It is immediate from the definition of $T_{\phi}$ that the map $\phi \rightarrow T_{\phi}$ is *-linear and contractive, that $T_{\phi} T_{\psi}=T_{\phi \psi}$ for $\phi$ in $L^{\infty}(m)$ and $\psi$ in $H^{\infty}$, and finally, as a consequence of Lemma 2, that $\left\|T_{\phi}\right\|=\|\phi\|$ for $\phi$ in $H^{\infty}$. For $B$ a closed subalgebra of $L^{\infty}(m)$, let $J_{B}$ be the smallest closed algebra of operators on $H^{2}$ containing $\left\{T_{\phi}: \phi \in B\right\}$ and let $\mathfrak{C}_{B}$ be the smallest closed ideal in $\Im_{B}$ containing $\left\{T_{\phi} T_{\psi}-T_{\phi \psi}: \phi, \psi \in B\right\}$. If $\Lambda$ is the map from $B$ to $\mathfrak{J}_{B} / \mathfrak{C}_{B}$ defined by $\Lambda(\phi)=T_{\phi}+\mathfrak{e}_{B}$ then $\Lambda$ is surjective, contractive, linear, and multiplicative. If $\Lambda$ were, in addition, isometric, then $\Lambda$ would be an isomorphism between $B$ and $J_{B} / \mathcal{C}_{B}$ and properties of the operators $T_{\phi}$ could be verified by considering the functions $\phi$ and the ideal $\mathfrak{C}_{B}$. The purpose of this section is to establish a condition on $B$ which makes $\Lambda$ isometric.

Set $R_{B}=B \cap H^{\infty}$ and let $S_{B}$ be the semigroup of functions $\psi$ in $R_{B}$ such that $\psi^{-1}$ is in $B$. If we set $Q_{B}=\left\{\phi / \psi: \phi \in R_{B}, \psi \in S_{B}\right\}$ then $Q_{B}$ is a subalgebra of $B$.

THEOREM 1. If $Q_{B}$ is dense in $B$, then $\Lambda$ is isometric.

Proof. Suppose $Q_{B}$ is dense in $B$. Let $w$ be the smallest subalgebra of $J_{B}$ containing $\left\{T_{\phi}: \phi \in Q_{B}\right\}$ and let $\mathcal{C}_{\mathfrak{W}}$ be the smallest ideal of $w$ containing $\left\{T_{\phi} T_{\psi}-T_{\phi \psi}: \phi, \psi \in Q_{B}\right\}$. Since $Q_{B}$ is dense in $B$, it suffices to show

$$
\|T \phi+V\| \geqq\|\phi\| \text { for } \phi \text { in } Q_{B} \text { and } V \text { in } \mathcal{C}_{w} .
$$

But if $\phi$ is in $Q_{B}$ and $V$ is in $\mathcal{C}_{w}$ there is a $\psi$ in $S_{B}$ such that $\left(T_{\phi}+V\right) T_{\psi}$ $=T_{\phi \psi}$ and $\phi \psi$ is in $H^{\infty}$. Thus, for any $\theta$ in $H^{\infty}$ with $\|\theta \psi\|>0$,

$$
\left\|T_{\phi}+V\right\| \geqq \frac{\|\phi \theta \psi\|}{\|\theta \psi\|}
$$

Let $\epsilon>0$ and let $U=\{y \in Y:|\hat{\phi}(y)|>\|\phi\|-\epsilon\}$. By Lemma 1 there is a $\theta$ in $H^{\infty}$ such that $\|\theta \psi\|>0$ and $\hat{\theta} \hat{\psi}$ peaks on $U$. Hence

proving $(*)$.

$$
\left\|T_{\phi}+V\right\| \geqq \frac{(\|\phi\|-\epsilon)\|\theta \psi\|}{\|\phi \psi\|}=\|\phi\|-\epsilon
$$


4. Applications. In this section the isomorphism theorem is applied to the algcbras $B=L^{\infty}(m)$ and $B=C$. That $Q_{L^{\infty}(m)}$ is dense in $L^{\infty}(m)$ is a consequence of the theorem of Tumarkin and Havinson stated in $\$ 1$; the argument is due to W. Rudin. That $Q_{c}$ is dense in $C$ is the Hartogs-Rosenthal theorem on rational approximation [8, p. 47]. The isomorphism theorem says that

$$
L^{\infty}(m) \stackrel{\Lambda_{L_{\infty}}}{\approx} \Im_{L^{\infty}} / \mathfrak{C}_{L^{\infty}} \text { and } C \stackrel{\Lambda_{C}}{\approx} \Im_{C} / \mathfrak{C}_{C} .
$$

Theorems 2 and 3 below are consequences of these isomorphisms and the fact that $\mathfrak{C}_{C}$ is the closed ideal $\mathcal{K}$ of compact operators.

If $\mathscr{L}$ is the Banach algebra of bounded operators on $H^{2}$, then an operator $T$ in $\mathscr{L}$ is Fredholm if $T+\mathfrak{K}$ is invertible in $\mathscr{L} / \mathfrak{K}$ : this is equivalent to saying that the range of $T$ is closed and the dimensions of the kernels of $T$ and $T^{*}$ are finite [5, Chapter 5]. The index of a Fredholm operator $T$ is defined to be $\operatorname{Ind}(T)=\operatorname{dim} \operatorname{ker} T-\operatorname{dim} \operatorname{ker} T^{*}$. Let $\sigma(T)$ denote the spectrum of $T$ and let $\sigma_{e}(T)$ be its essential spectrum-the spectrum of $T+\mathcal{K}$ in $\mathcal{L} / \mathcal{K}$. For a function $\phi$ in $L^{\infty}(m)$ let $R(\phi)$ be its essential range.

THEOREM 2. If $\phi$ is in $L^{\infty}(m)$, then $R(\phi) \subset \sigma_{\theta}\left(T_{\phi}\right) \subset \sigma\left(T_{\phi}\right)$ Cconvex hull $R(\phi)$ and thus $\left\|T_{\phi}\right\|=\|\phi\|$.

TheOREM 3. If $\phi$ is in $C$, then $\sigma_{e}\left(T_{\phi}\right)=R(\phi)$ and for $\lambda$ not in $R(\phi)$ $\operatorname{Ind}\left(T_{\phi}-\lambda\right)=-\operatorname{Ind}(\phi, \lambda)$.

In computing Ind $(\phi, \lambda)$, the boundary of $D$ is oriented positively.

5. The algebra $H^{\infty}+C$. Let $H^{\infty}+C$ be the set of functions in $L^{\infty}(m)$ of the form $\phi+f$ where $\phi$ is in $H^{\infty}$ and $f$ is in $C . H^{\infty}+C$ is then a closed subalgebra of $L^{\infty}(m)$ (cf. [14]). For a function $\phi$ in $L^{\infty}(m)$ let $\tilde{\phi}$ denote its harmonic extension into $D$.

THEOREM 4. For $\phi$ in $H^{\infty}+C$, the following statements are equivalent:

(i) $T_{\phi}$ is Fredholm.

(ii) $\phi$ is invertible in $H^{\infty}+C$.

(iii) There is a compact set $K$ in $D$ and an $\epsilon>0$ such that

$$
|\tilde{\phi}(z)| \geqq \epsilon \text { for } z \text { in } D \backslash K \text {. }
$$

If these conditions are satisfied, then $\operatorname{Ind}\left(T_{\phi}\right)=-\operatorname{Ind}(\tilde{\phi} \circ \sigma, 0)$ where $\sigma$ is a cycle in $D \backslash K$ homotopic in $\bar{D} \backslash K$ to the positively oriented boundary of $D$.

PROOF. The proof of the equivalence of (ii) and (iii) is similar to the proof for the disk [4], [5, Chapter 6]. That (ii) implies (i) is a consequence of the isomorphism theorem. To show that (i) implies (ii), 
it is sufficient to consider the case $\phi$ in $H^{\infty}$ (cf. [4]). In this case, one lif ts the function $\phi$ to a function $\Phi$ on the disk by means of a covering map. $\Phi$ is then factored $\Phi=\psi F$ where $\psi$ is inner and $F$ is outer; these factors are modulus automorphic [11]. The assumption that $T_{\phi}$ is Fredholm implies that $\psi$ is a Blaschke product for finitely many points in $D$. One then produces an outer function $G$ in $H^{\infty}$ of the disk which is bounded below and modulus automorphic such that $\psi / G$ and $G F$ are automorphic. Moreover, the function $\psi / G$ is the lift of a continuous function on $\bar{D}$. Since $\Phi=(F G)(\psi / G), \phi$ is invertible in $H^{\infty}+C$. The statement about the index of $T_{\phi}$ is verified for $\phi$ in $H^{\infty}$ by showing $\operatorname{Ind}\left(T_{\phi}\right)=-$ numbers of zeros of $\phi$; the statement for general $\phi$ in $H^{\infty}+C$ is a consequence of this and Theorem 3.

6. Reduction to Toeplitz operators on the disk. Let $B_{0}, \cdots, B_{n}$ be the components of the boundary of $D$ with $B_{0}$ forming the outer boundary. Let $D_{0}$ be the interior of $B_{0}$ and $D_{1}, \cdots, D_{n}$ be the exteriors of $B_{1}, \cdots, B_{n}$ including the point at infinity. By the Riemann mapping theorem there is a conformal equivalence $\Pi_{i}$ from the open unit disk $\Delta$ to $D_{i}$ and since the boundary of $D$ is analytic $\Pi_{i}$ extends to a homeomorphism from $\bar{\Delta}$ to $B_{i} \cup D_{i}$. Associated with a function $\phi$ in $L^{\infty}(m)$ are the functions $\phi_{i}=\phi \circ \Pi_{i}$ in $L^{\infty}(\partial \Delta)$. The following theorem relates to Toeplitz operator $T_{\phi}$ with the Toeplitz operators $T_{\phi_{i}}$ on $H^{2}$ of the disk.

THEOREM 5. $T_{\phi}$ is Fredholm if and only if $T_{\phi_{i}}$ is Fredholm for $i=0,1, \cdots, n$ and thus $\sigma_{e}\left(T_{\phi}\right)=\bigcup_{i=0}^{n} \sigma_{e}\left(T_{\phi_{i}}\right)$. If $T_{\phi}$ is Fredholm then $\operatorname{Ind}\left(T_{\phi}\right)=\sum_{i=0}^{n} \operatorname{Ind}\left(T_{\phi_{i}}\right)$.

Theorem 5 has two interesting corollaries. The first is a consequence of a recent result due to Douglas and Sarason which says that for a Toeplitz operator on the disk, the property of being Fredholm is a local property [7]. This says that if $\phi$ "looks Fredholm" in a neighborhood of each point on the circle, then $T_{\phi}$ is Fredholm. Theorem 5 extends this result to multiply connected regions. Furthermore, it shows that the local property involved is the same.

The second corollary stems from Widom's proof that the spectrum of a Toeplitz operator on the disk is connected [13]. With minor modifications [5, Chapter 6] this proof shows that the essential spectrum is also connected. From Theorem 5 we may conclude that the essential spectrum of a Toeplitz operator on an $n$-holed region has at most $n+1$ components.

7. Remarks. Let $\phi$ be the characteristic function of a component of the boundary of $D$. Then $T_{\phi}$ is selfadjoint, $\sigma_{\bullet}\left(T_{\phi}\right)=\{0,1\}$, and 
0 and 1 are not eigenvalues of $T_{\phi}$ (for this would imply that a nonzero function in $H^{\perp \perp}$ could vanish on a set of positive measure, an impossibility). Thus, the spectrum of $T_{\phi}$ consists of $\{0,1\}$ and eigenvalues of finite multiplicity in $(0,1)$ which accumulate at 0 and 1 . This provides an example asked for in [6] of a generalized Toeplitz operator with disconnected spectrum. The example shows that the nature of the spectrum of a Toeplitz operator is considerably different when the domain $D$ is not simply connected. Two reasons for this are suggested below.

A lemma due to Coburn [2] says that a nonzero Toeplitz operator on the disk has trivial kernel or trivial cokernel. Thus, the spectrum is obtained from the essential spectrum by filling in the components of the essential resolvent which have nonzero index. The lemma is proved in the following way. If $T_{\phi}(f)=0$ and $T_{\phi}^{*}(g)=0$ then $\phi f \bar{g}$ is in $N$, the $L^{1}$-annihilator of $A+\bar{A}$. In the disk, $\operatorname{dim} N=0$ and the lemma follows from the F. and M. Reisz Theorem. In an $n$-holed region $\operatorname{dim} N$ $=n$. Thus, it is possible to have a Fredholm Toeplitz operator on a multiply connected region with zero index which is not invertible.

A second difference between the disk and the multiply connected case is the appearance of important spaces of multiple-valued functions. For each character $\alpha$ on the first homology group $G$ of $D$, there is associated a Hilbert space $H_{\alpha}^{2}$. Elements of $H_{\alpha}^{2}$ may be thought of as functions on the disk which are modulus automorphic [11] or they may be thought of as certain functions on $D \times G$ [9]. For each $\phi$ in $L^{\infty}(m)$ and each character $\alpha$ there is a Toeplitz operator $T_{\phi}^{\alpha}$ on $H_{\alpha^{*}}^{2}$ In some cases, the proper generalization of a theorem about a Toeplitz operator $T_{\phi}$ on the disk is a theorem about the entire bundle of operators $\left\{T_{\phi}^{\alpha}\right\}$. For instance, for a unimodular function $\phi$ on the unit circle, $T_{\phi}$ is left invertible if and only if the distance from $\phi$ to $H^{\infty}$ is less than 1 [12]. In a more general region we have

THEOREM 6. Suppose $|\phi|=1$ a.e. Then $T_{\phi}^{\alpha}$ is left invertible for each $\alpha$ if and only if the distance from $\phi$ to $H^{\infty}$ is less than 1.

\section{BIBLIOGRAPHY}

1. P. R. Ahern and D. Sarason, The $H^{p}$ spaces of a class of function algebras, Acta. Math. 117 (1967), 123-163. MR 36 \#689.

2. L. A. Coburn, Weyl's theorem for nonnormal operators, Michigan Math. J. 13 (1966), 285-288. MR 34 \#1846.

3. R. G. Douglas, On the spectrum of Toeplitz and Wiener-Hopf operators, Proc. Conference on Abstract Spaces and Approximation (Oberwohlfach, 1968), Internat. Schriftenreihe Numer. Math., vol. 10, Birkhäuser Verlag, Basel, 1969.

4. - Toeplitz and Wiener-Hopf operators in $H^{\infty}+C$, Bull. Amer. Math. Soc. 74 (1968), 895-899. MR $37 \# 4648$. 
5. - Topics in analysis, Holt, Rinehart and Winston, New York, N. Y., 1971 (to appear).

6. R. G. Douglas and C. Pearcy, Spectral theory of generalized Toeplitz operators, Trans. Amer. Math. Soc. 115 (1965), 433-444. MR 33 \#7849.

7. R. G. Douglas and D. Sarason, Fredholm Toeplitz operators, Proc. Amer. Math. Soc. 26 (1970), 117-120.

8. T. Gamelin, Uniform algebras, Prentice-Hall, Englewood Cliffs, N. J., 1969.

9. M. Hasumi, Invariant subspaces for finite Riemann surfaces, Canad. J. Math. 18 (1966), 240-255. MR 32 \#8200.

10. G. C. Tumarkin and S. Ya. Havinson, On the existence in multiply-connected regions of single-valued analytic functions with a given modulus of boundary values, Izv. Akad. Nauk SSSR Ser. Mat. 22 (1958), 534-562. (Russian) MR 20 \#3990.

11. M. Voichick and L. Zalcman, Inner and outer functions on Riemann surfaces, Proc. Amer. Math. Soc. 16 (1965), 1200-1204. MR 32 \#1359.

12. H. Widom, Inversion of Toeplitz matrices. III, Notices Amer. Math. Soc. 7 (1960), 63. Abstract \#564-246.

13. - Toeplitz operators on $H_{p}$, Pacific J. Math. 19 (1966), 573-582. MR 34 \#1859.

14. L. Zalcman, Bounded analytic functions on domains of infinite connectivity, Trans. Amer. Math. Soc. 144 (1969), 241-269.

University of Michigan, ANn Arbor, Michigan 48104

State University of New York, Stony Brook, New York 11790 